\title{
Entrevista a Abraham Magendzo Kolstrein sobre educación inclusiva y la enseñanza de los derechos humanos ${ }^{1}$
}

\author{
Abraham Magendzo Kolstrein entrevistado por Francisco Gárate, ${ }^{2}$ Concepción López-Andrada, ${ }^{3}$ \\ Aldo Ocampo-González $z^{4}$ L Luis Pincheira
}

Resumen: al abordar la enseñanza de los derechos humanos en el contexto de América Latina es necesario situar como figura relevante al profesor Abraham Magendzo. Durante esta conversación caracterizada por el análisis del presente, pero también por la memoria y el recorrido histórico de la educación en derechos humanos especialmente en Chile, se destaca la dificultad a la hora de desarrollar proyectos educativos en los que toda la comunidad educativa tome consciencia de la importancia de la materia en derechos humanos. El elemento interdisciplinar y transversal debe ser el motor que canalice estas propuestas desde y para la democracia y la inclusión. El entrevistador se cuestiona de forma principal, acerca de cómo se ha asumido que la educación en derechos humanos debe ser una formación limitada a los sufrientes u oprimidos, cuando debería ser un proceso que alcanzara a toda la ciudadanía. Asimismo, la

\footnotetext{
${ }^{1}$ Entrevista efectuada el día o3 de julio de 2019 como parte del ciclo de entrevistas a destacadas personalidades de las Ciencias Sociales, el pensamiento crítico y la educación, organizado por el Centro de Estudios Latinoamericanos de Educación Inclusiva, primer centro de investigación creado en Alac y Chile, para el estudio teórico y metodológico de la Educación Inclusiva. Centro miembro Clacso.

${ }^{2}$ Chileno. Doctor en Planificación Educativa e Innovación Educativa (UAH, España). Investigador Centro de Estudios Latinoamericanos de Educación Inclusiva, Chile.

3 Española. Doctora en Educación (UEX, España). Directora del Observatorio sobre Educación Lectora, Nuevas Ciudadanías y Democracia, Centro de Estudios Latinoamericanos de Educación Inclusiva, Chile.

4 Chileno. Doctor en Ciencias de la Educación, Sobresaliente Cum Laude (UGR, España). Director fundador Centro de Estudios Latinoamericanos de Educación Inclusiva, Chile. Teórico y crítico educativo.

5 Chileno. Doctor en Educación (UAHC, Chile). Centro de Estudios Latinoamericanos de Educación Inclusiva, Chile.
} 
enseñanza y aprendizaje en Derechos humanos según Magendzo, debería empezar desde edades muy tempranas en los estudiantes, ya que estos están asociados a comportamientos que se reflejarán en las aulas. Finalmente, los desafíos que propone el entrevistado pasan por la formación del profesorado, el compromiso y la búsqueda constante de la rigurosidad en la materia.

Palabras clave: derechos humanos, América Latina, educación inclusiva, ciudadanía.

Abstract: When approaching the teaching of Human Rights in the context of Latin America, it is necessary to situate Professor Abraham Magendzo as a relevant figure. During this conversation characterized by the analysis of the present, but also by the memory and the historical journey of Human Rights education, especially in Chile, the difficulty in developing educational projects in which the entire educational community becomes aware of the importance of the subject in Human Rights is highlighted. The interdisciplinary and transversal element must be the motor that channels these proposals from and for democracy and inclusion. The interviewer mainly questions how it has been assumed that education in human rights should be limited to the suffering or oppressed, when it should be a process that reaches all citizens. Likewise, teaching and learning in Human Rights according to Magendzo, should start from very early ages in students, since these are associated with behaviors that will be reflected in the classrooms. Finally, the challenges proposed by the interviewee include teacher training, commitment and the constant search for rigor in the matter.

Keywords: Human Rights, Latin America, inclusive education, citizenship.

\section{Bionota del entrevistado}

Abraham Magendzo Kolstrein. Premio Nacional de Educación de Chile, 2017. Profesor de Estado en Educación y Orientador Educacional de la Universidad de Chile. Máster en Educación e Historia de la Universidad Hebrea (Israel) y doctor en Educación de la Universidad de California (Estados Unidos). También posee un posdoctorado en Currículo, realizado en el Instituto de Educación de la Universidad de Londres (Inglaterra).

Tiene más de 40 años de experiencia laboral en el plano educativo, desempeñándose en distintos cargos académicos, entre los que se encuentran el de profesor titular del Departamento de Currículum de la Pontificia Universidad Católica de Chile; rector del Instituto Hebreo y director ejecutivo del Programa Interdisciplinario de Investigaciones en Educación (PIIE), donde también ha trabajado como investigador, función que también ha desempeñado 
en la Fundación Ideas de Chile. También ha integrado la Unidad de Currículum y Evaluación del Ministerio de Educación, donde se desempeñó como coordinador de los Objetivos Fundamentales Transversales.

Desde 1996 se desempeña como profesor e investigador de currículo en la Universidad Academia de Humanismo Cristiano, donde también es director académico del Programa de Doctorado en Educación y coordinador de la Cátedra Unesco de Educación en Derechos Humanos. Además, a partir de 2015, es parte del Consejo Consultivo del Instituto Nacional de Derechos Humanos y ha sido consultor internacional y conferencista de Unesco, la OEA, el Instituto Interamericano de Derechos Humanos, la Fundación

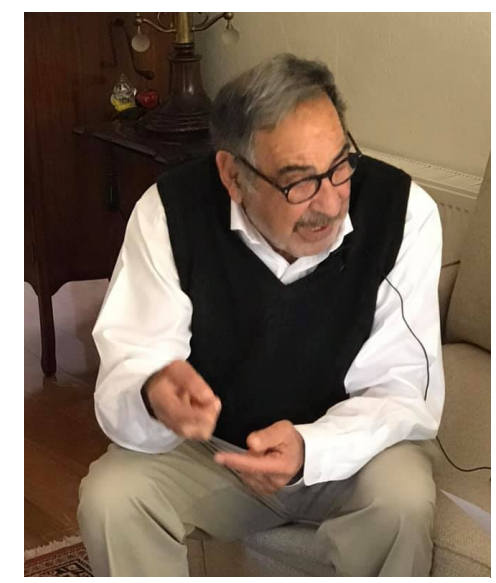

Fotografía tomada el día miércoles o3 de julio de 2019, con motivo de la celebración de la entrevista. Autorizada por el entrevistado. Ford y el Instituto para la Democracia Luis Carlos Galán de Colombia. Mantiene relaciones académicas con muchas universidades y centros de estudios en América Latina y Europa.

Es autor de alrededor de 7o libros, publicaciones y artículos académicos. Entre las distinciones que ha recibido destacan la Orden al Mérito Gabriela Mistral (2004), otorgada por el Gobierno de Chile a través del Ministerio de Educación, por su contribución en beneficio de la educación, la cultura y el enaltecimiento de la función docente.

Luis Pincheira (L. P.): En primer lugar, agradecerle desde el Centro de Estudios Latinoamericanos de Educación Inclusiva (Celei), su tiempo con nosotros, con el objeto de abordar un conjunto de cuestiones relacionadas con la educación y los derechos humanos.

Abraham Magendzo (A. M.): Gracias a ustedes por invitarme a conversar sobre estos temas que hemos trabajado en la cátedra de Unesco; existe una red completa donde convergen muchas instituciones. Entonces, yo agradezco que me hayan invitado a conversar.

L. P.: Quisiéramos conocer su opinión sobre la educación de los derechos humanos en la actualidad en América Latina y específicamente en Chile.

A. M.: La pregunta que tú me haces me motiva y me induce a hacer un poco de historia sobre la educación en derechos humanos en América latina. Uno podría decir (creo no equivocarme) dos cosas: uno, que nosotros hicimos conciencia, no solamente en Chile sino en muchos países de Latinoamérica que, diría yo casi la mayoría. Como resultado de la dictadura que nuestros países vivieron y la lucha que se hizo para recuperar la democracia, la primera pregunta que surgió fue, ¿bueno y ahora qué? Si durante esta dictadura se violaron los derechos huma- 
nos, la lógica indicaba obviamente que lo primero que había qué hacer es educar en derechos humanos, para nunca más, para que esto no volviera a suceder en Chile nuevamente y en América Latina también.

Yo tengo siempre una anécdota que cuento en relación con esta disyuntiva de recuperar la democracia, que el centro de la educación fuera la educación en derechos humanos para un nunca más. Te tengo una pequeña historia de una persona que iba a ser nombrada, recuperada la democracia, en el gobierno de Aylwin ${ }^{6}$ iba a ser nombrada ministro de educación. Entonces me llamó - yo lo conocía- y entonces estuvimos conversando y me dijo: mira Abraham, este asunto que tú estás insistiendo tanto de que se pusiera en el currículum una asignatura o un curso sobre educación en derechos humanos... tú sabes está todavía Pinochet aquí; está la derecha dura todavía presente que apoyó a la dictadura. Entonces, en vez de hablar de educación en derechos humanos hablemos de educación para el amor. Entonces, yo lo miré y le dije: mira, yo personalmente no tengo nada en contra del amor, muy por el contrario, pero son cosas distintas. El lenguaje crea realidad; el lenguaje está conectado con una realidad social, histórica. De este modo, lo que quiero señalar es que cuando con nosotros ya a finales de la década de los ochenta y comienzo de los noventa (no solamente en Chile sino que en toda América Latina), realmente el recorrido que teníamos en relación con la educación en derechos humanos era muy escuálido, no existía por así decirlo, no habíamos hecho nada al respecto. Tomamos conciencia de eso como producto de las violaciones de derechos humanos que sucedieron en nuestros países y, entonces, realmente, el primer país que comenzó a preparar profesores en educación de derechos humanos fue Uruguay. Serpaj’ empezó todavía cuando estábamos en Chile en dictadura, empezó a hacer cursos de educación en derechos humanos; pero los cursos eran como muy legalistas, te enseñaban qué son los derechos humanos, la declaración universal, que es lo que significa, no la violación de los derechos humanos. Todo era muy normativo y muy escuálido.

En Chile me convidó un buen amigo mío, Jorge Osorio, ${ }^{8}$ a dictar unas charlas sobre educación en derechos humanos a gente que había sufrido la represión aquí en Chile. Era todavía en el tiempo de la dictadura. Entonces, yo escuché a la gente que había sufrido tanto, y yo en-

6 Patricio Aylwin Azocar (1919-2016). Político y abogado chileno. Presidente de la República (1990-1994) posdictadura cívico militar de Augusto Pinochet Ugarte.

7 Servicio de Paz y Justicia. Corporación en su mayoría conformada por jóvenes cristianos de diversas tendencias políticas que, durante el período de la dictadura militar, denunciaron las violaciones de los derechos humanos, mediante acciones públicas, jornadas de entrenamiento, escuelas de no violencia y talleres de control del miedo.

8 Jorge Osorio Vargas. Historiador, licenciado en Historia en la PUCV. Estudios de postgrado en Derechos Humanos y Desarrollo en el ISS de La Haya. Docente de la Facultad de Educación de la Universidad de Playa Ancha y de la Escuela de Psicología de la Universidad de Valparaíso. 
tonces ahí me pregunté: ¿por qué la educación en derechos humanos tiene qué hacerse exclusivamente con los sufrientes, con los que habían sufrido la represión en carne propia?, porque ahí estaban reunidos en Punta de Tralca. Ahí comencé y comenzamos en el PIIE9 a pensar en capacitar profesores para la educación en derechos humanos. Hicimos cursos en el CPEIP, ${ }^{10}$ hicimos unos, dictamos unos cursos a profesores en educación en derechos humanos y empezamos a escribir también, empezamos a investigar. Pero esto no solamente en Chile; esto se empezó a hacer en Argentina, se empezó a hacer en Brasil, se empezó a hacer en Perú y grandes educadores que empezaron a trabajar el tema de la educación en derechos humanos en América Latina.

Y hoy día debo señalar, diría yo con mucho orgullo y también con mucha satisfacción, que hemos avanzado bastante, pero no lo suficiente, no lo suficiente. Voy a explicar a propósito de la pregunta relacionada con la educación superior, no lo suficiente, pero hemos avanzado. Hoy día tenemos entre otras cosas, tenemos materiales sobre educación en derechos humanos, revistas sobre educación en derechos humanos (en casi todos los países), además de la red latinoamericana y del Caribe de educadores y de educación, y de educadores en derechos humanos; tenemos una red, nos vamos a reunir este año en octubre en Antofagasta, van a venir de toda América Latina y del Caribe a reunirse. No es la primera vez que nos reunimos, nos vamos a reunir la red. Entonces hoy día tenemos redes, red latinoamericana, cada país tiene una red, nosotros en Chile tenemos una red de instituciones que están preocupadas de la educación en derechos humanos. Entonces yo podría decir que hemos avanzado enormemente, pero el camino que tenemos por delante todavía es muy extenso, muy largo.

Concepción López-Andrada (C. L. A.): ¿Cómo podemos avanzar para construir una ciudadanía que sea crítica y, a la vez, que no sea excluyente?

A. M.: Todos estos temas son temas de alta complejidad. A veces caemos en el error $-\mathrm{y}$ yo también me sumo a eso- que creemos que con una conferencia vamos a dar respuesta a la pregunta que estás haciendo. Entonces, son procesos largos. Acabo de escribir un libro con un amigo, Jorge Pavés, sobre educación en derechos humanos; lo publicó el año pasado Santillana y llama Formación ciudadana y temas controversiales; sobre temas controversiales voy a conver-

9 Programa Interdisciplinario de Investigación. Corporación de derecho privado sin fines de lucro, con la calidad de organización no gubernamental y centro académico independiente. La gestación del PIIE tiene lugar hacia fines de los años 60 .

${ }^{10}$ Centro de Perfeccionamiento, Experimentación e Investigaciones Pedagógicas. Organismo del Ministerio de Educación de Chile encargado de implementar y ejecutar la ley que crea el Sistema de Desarrollo Profesional Docente, la cual define las bases de una política pública que potencia, orienta y regula el desarrollo de docentes y educadores. 
sarte más adelante, pero la formación ciudadana es vital para la inclusión, vale decir, cómo la inclusión entrega un mensaje, no solamente de derechos humanos sino un mensaje ciudadano, central. Nosotros estamos viviendo en este país - y en otros también en América Latina- con una sociedad muy segmentada; se juntaron todos los planos: en el plano económico, en el plano social, en el plano de las viviendas. Yo vivo gracias a dios en un barrio muy acomodado de nuestra ciudad, pero mis nietos no salen de aquí; entonces vivimos en una sociedad muy segmentada y vivimos una educación muy segmentada. ¿Cómo formamos ciudadanos si vivimos en verdaderos guetos?, ¿cómo aprendemos en una sociedad? Entonces, la educación tiene un rol fundamental que jugar.

L. P.: Con el propósito de construir, como dice usted, inclusión, justicia y lograr esto en la sociedad, ¿qué es lo que necesitamos realmente?

A. M.: La formación, desafortunadamente, y nosotros hemos hecho investigaciones donde se muestra claramente que con toda la voluntad que puedan tener los profesores para enseñar estos temas, para enseñar el tema ciudadano, el tema, no tienen una práctica construida. Nadie, si tú vas a contar a propósito de la red chilena, las instituciones formadoras de maestros en este país, formadores de profesores y profesoras en este país que dictan algún curso o una cátedra sobre derechos humanos, yo te los puedo contar con los dedos de la mano, y quizás me sobrarían dedos. Es decir, entonces, y cuando se les larga a los profesores, se les larga digo, cuando se le entrega a los profesores esta misión, ellos no saben qué hacer. Hemos investigado eso. No están preparados; porque están preparados en las matemáticas. Entonces ahí tienes otro tema, ahí tienes otro tema que está ligado a la educación superior.

Nuestra postura ha sido y es que los derechos humanos no son la enseñanza de los derechos humanos y de la ciudadanía; no es propiedad del profesor de historia o del profesor de filosofía, sino que son completamente transversales; y el ingeniero en la escuela de ingeniería tendría que entender, el arquitecto tendría qué entender que construir una ciudad segregada y urbanizada de esa manera, con edificios uno encima de otro y la gente lo único que ve es cemento. Tú te das cuenta que no hay ninguna sensibilidad por parte de la educación superior, no hay sensibilidad de que ellos sean responsables de educar en derechos humanos también. Mira, no hablo del filósofo, del historiador —eso no cabe la menor duda-, pero el médico, el dentista... Alguien me hacía una broma, que el dentista es el gran violador de derechos humanos porque le pone un algodón en la boca y no lo deja hablar y él habla y uno no tiene el derecho a la libre expresión. Bueno, eso es un chiste, pero todos los profesores tendrían que tener sensibilidad para el tema de los derechos humanos. Mira tú cómo se construyen nuestras ciudades, cómo se desperdicia agua, cómo violamos el medio ambiente, todos esos son derechos. 
En medio ambiente hoy existe un derecho al medio ambiente, a una ecología sustentable; así, cuando tú me preguntas sobre cuáles son las deficiencias que hay, es que este país y quizás no solamente este país, no ha hecho conciencia que a sus profesionales - y hablamos de los profesionales yo diría en general-, pero los profesionales tengan sensibilidad frente al tema y cómo ellos pueden contribuir a sensibilizar a la sociedad en su conjunto sobre los derechos humanos. No solamente se violaron los derechos humanos durante la dictadura, ciertamente que se violaron, pero estamos hoy día violando derechos fundamentales.

Francisco Gárate (F.G.): Frente a esta realidad que usted nos expone, ¿por qué es importante mirar hacia el futuro, no tan a futuro sino que ahora en el presente, a aquellas generaciones jóvenes?, y ¿qué elementos pueden estructurar la enseñanza de estos derechos en su formación como futuros ciudadanos?

A. M.: Pienso que la enseñanza y el aprendizaje, y el comportamiento, digamos, relacionado con los derechos humanos debiera comenzar desde edades muy tempranas; y hay experiencias, hay materiales que muestran esto. No hay que inventar el agua caliente, digamos. Hay materiales, hay que ponerlos en práctica, hay pensamiento en relación con la educación en derechos humanos desde edades muy tempranas, desde edades muy tempranas. Todo el tema que yo he trabajado también sobre el bullying; el bullying es una violación a derechos humanos tremenda. Yo iba a escribir un artículo (después no lo escribí) sobre comparar a los violadores de derechos humanos durante la dictadura y a los que cometen bullying en las escuelas; después no lo hice. Pero hay elementos, si miras a un bulero, a un intimidador, su comportamiento, es un comportamiento violador de derechos humanos básico, en la falta de empatía, de respeto hacia el otro y la otra, distinto, entonces, esto hay que comenzarlo de edades muy tempranas.

Aldo Ocampo González (A. O. G.): Si bien es cierto, como usted dice, hay material, existen diversos insumos, entonces, ¿de qué manera se debiese abordar la enseñanza de los derechos humanos en la formación de los futuros educadores?

A. M.: Exactamente, yo creo que tal como te decía son contadas las universidades e inclusive las instituciones formadoras de maestros que tengan cursos o cátedras relacionadas; estoy hablando de Chile, y podría hasta probarlo a muchos otros países. En Argentina han progresado mucho al respecto; los argentinos han trabajado muchísimo y tienen cursos y tienen formación en las instituciones formadoras de maestros, cosa que nosotros no hemos hecho. Entonces deberíamos intervenir en las instituciones formadoras de maestros. Ahora quieren crear $-\mathrm{y}$ yo he estado al pendiente- un curso en tercero y cuarto medio, una asignatura de formación ciudadana donde los derechos humanos son centrales. Si no se respetan los derechos del otro, los míos y los del otro y otras, ¿de qué formación ciudadana estamos hablando? Entonces ese curso 
o esa disciplina que se va a dictar va a estar muy ligada a los derechos humanos y, en consecuencia las instituciones formadoras de maestros, formadoras de profesores, van a tener qué incorporar estos temas en su formación; porque si en tercero y cuarto medio va haber una asignatura llamada Formación ciudadana y los jóvenes (los profesores jóvenes) no se preparan para enseñar esto, es inconducente.

F. G.: Antes de finalizar, le agradecemos su tiempo, nos quedamos impregnados de su sabiduría en este tema. Sin embargo, ¿qué desafíos o recomendaciones podría hacernos ya sobre este tema que hoy día nos convoca?

A. M.: El desafío es —cómo decirlo- tomárselo en serio. Yo creo que en educación tenemos que tomarnos en serio la cosa. Hay voluntad (es lo que te decía anteriormente) de parte de los profesores y profesoras de enseñar esto. Yo creo que hoy día no es que haya reticencia de decir, esto no es mío, esto no; hay voluntad, pero no hay preparación.

L. P.: La pregunta es, ¿quién se hace cargo, cierto?

A. M.: Claro, pero mira, es que algo yo te decía que son los profesores y las profesoras, pero son otros profesionales también. Entonces, brevemente, hay que empezar a educar a profesionales a que tomen conciencia de que ellos tienen responsabilidad frente al tema de los derechos humanos.

L. P.: Querido Abraham, queremos agradecerte por tu tiempo, sabemos que podríamos estar hablando todo un día. Me imagino que este tema te fascina, te convoca; pero dado tu tiempo, queremos volver a agradecerte como Celei esta entrevista, esperando que en una próxima oportunidad nos puedas nuevamente entregar una parte de tu tiempo.

A. M.: No, yo te agradezco a ti la oportunidad que me has dado y con gusto a futuro voy a poder colaborar con ustedes cien por ciento. 